\title{
Collinear masking effect in visual search is independent of perceptual salience
}

\author{
Li Jingling ${ }^{1,2}$ • Yi-Hui Lu ${ }^{1}$ • Miao Cheng ${ }^{3}$ - Chia-huei Tseng ${ }^{4}$
}

Published online: 23 March 2017

(C) The Psychonomic Society, Inc. 2017

\begin{abstract}
Searching for a target in a salient region should be easier than looking for one in a nonsalient region. However, we previously discovered a contradictory phenomenon in which a local target in a salient structure was more difficult to find than one in the background. The salient structure was constructed of orientation singletons aligned to each other to form a collinear structure. In the present study, we undertake to determine whether such a masking effect was a result of salience competition between a global structure and the local target. In the first 3 experiments, we increased the salience value of the local target with the hope of adding to its competitive advantage and eventually eliminating the masking effect; nevertheless, the masking effect persisted. In an additional 2 experiments, we reduced salience of the global collinear structure by altering the orientation of the background bars and the masking effect still emerged. Our salience manipulations were validated by a controlled condition in which the global structure was grouped noncollinearly. In this case, local target salience increase (e.g., onset) or global distractor salience reduction (e.g., randomized flanking orientations) effectively removed the facilitation effect of the noncollinear structure. Our data suggest that salience competition is
\end{abstract}

Chia-huei Tseng

CH Tseng@alumni.uci.edu

1 Graduate Institute of Neural and Cognitive Sciences, China Medical University, Taichung, Taiwan

2 Graduate Institute of Biomedical Sciences, China Medical University, Taichung, Taiwan

3 Department of Psychology, University of Hong Kong, Pok Fu Lam, Hong Kong

4 Research Institute of Electrical Communication, Tohoku University, Sendai, Japan unlikely to explain the collinear masking effect, and other mechanisms such as contour integration, border formation, or the crowding effect may be prospective candidates for further investigation.

Keywords Collinear grouping $\cdot$ Salience $\cdot$ Visual search $\cdot$ Attentional capture

Searching for a small item in a visually rich environment is a frequently encountered task in daily life (e.g., searching for a key in a room full of furniture and household clutter). Search efficiency is enhanced if the item (target) is salient (Wolfe, 2010). The term salience refers to the distinctiveness of an item from its neighbors in a physical dimension, such as color, luminance, shape, or movement (Itti \& Koch, 2001; Li, 2002; Wolfe, 2010; Zhaoping, 2014). If the target per se is not salient though overlapping with a salient region, we still benefit since a search for an overlapping target is more efficient than a search for a non-overlapping one (i.e., attention capture; Turatto \& Galfano, 2000, 2001; Turatto, Galfano, Gardini, $\&$ Mascetti, 2004). Among all forms of salience, abrupt onset is considered to be the most attractive property for attentional capture (Jonides \& Yantis, 1988; Rauschenberger, 2003; Theeuwes, 2010; Yantis \& Jonides, 1984).

\section{Effect of visual search target/distractor overlapping (1): Collinear masking}

Not all salient items inform visual searching. Our previous studies revealed a complex interaction between salience and perceptual grouping by demonstrating that some types of salient structures hinder search (Chiu \& Jingling, 2014, 2015; Chow, Jingling, \& Tseng, 2013, 2016; Jingling, Tan, \& Tseng, 
2013; Jingling \& Tseng, 2013; Jingling, Tseng, \& Zhaoping, 2013; Tseng \& Jingling, 2015). In these earlier studies, the search display was filled by horizontal bars, except for one salient and collinearly grouped column (Fig. 1a), the collinear distractor. A local target (a small, oriented black gap in Fig. 1) was designed to overlap with the collinear distractor occasionally (overlapping target). One would expect that an overlapping target would be easier to detect compared to a target placed on a background bar (nonoverlapping target), as it was in a salient region. Nevertheless, we found the opposite: an overlapping target was discriminated more slowly and less accurately than a nonoverlapping target, that is, there was a collinear masking effect.

\section{Effect of visual search target/distractor overlapping (2): Noncollinear facilitation}

A salient column constructed with bars forming a ladder structure as in Fig. 1b did not induce the masking effect. Instead, a target overlapping with this salient distractor became easier to locate (Jingling \& Tseng, 2013). Both the snake (see Fig. 1a) and ladder (Fig. 1b) structures had $90^{\circ}$ orientations in contrast to other background bars and were globally organized following a Gestalt grouping law of similarity. The snake distractor was additionally grouped by collinearity. In a visual search, the contrast between a masking effect induced by the collinear distractor and a facilitation effect induced by the non-collinear distractor indicates the involvement of a grouping mechanism that goes beyond a simple salience computation based on contrast of the basic features (Itti \& Koch, 2001).

\section{Attention and collinear grouping}

However, how such a masking effect is induced is still unclear. One possibility, which has not been critically tested, is that collinear grouping contributes to the computation of perceptual salience, and the salience competition between a global salient distractor and a less salient local target causes such a masking effect. This conjecture is supported by past studies demonstrating that collinear grouping improved visual contour detection (Hess, Hayes, \& Field, 2003; Vancleef \& Wagemans, 2013), discrimination (Dakin \& Baruch, 2009; Schmidt \& Vancleef, 2016; Vancleef \& Wagemans, 2013), and contour color change detection (Jingling \& Zhaoping, 2008) by strengthening perceptual salience. Also, collinearly grouped items facilitated target discrimination when the target was looked for inside the grouped objecthood (Conci, Müller, \& Elliott, 2007; Kimchi, Yeshurun, \& Cohen-Savransky, 2007; Kimchi, Yeshurun, Spehar, \& Pirkner, 2016), suggesting that collinear grouping has priority in attentional processing (Yeshurun, Kimchi, Sha'shoua, \& Carmel, 2009). Too strong collinear grouping can eliminate or interfere with memory-based contextual cuing effect by confining regional representation and setting priority in attentional processing (Conci, Müller, \& von Mühlenen, 2013; Conci \& von Mühlenen, 2009). In sum, collinearly grouped items capture attention better than ungrouped items. An eye-movement study showed that a target placed on the collinear distractor was perceptually less salient than a nonoverlapping target (Jingling, Tan, \& Tseng, 2013). In other words, it is possible that the collinear distractor in the search display in Fig. 1a was too salient so that the target lost its attentional priority and delayed the search compared to the target away from the collinear distractor (i.e., collinear masking effect). The goal of this study is to examine whether global and local salience competition account for this collinear masking effect.

\section{Design rationale}

We tested this salience competition hypothesis in two ways: by increasing the salience of the local target as well as by decreasing the salience of the global distractor. A similar design was applied in a global interference effect study (Han \&
(A) Collinear distractor

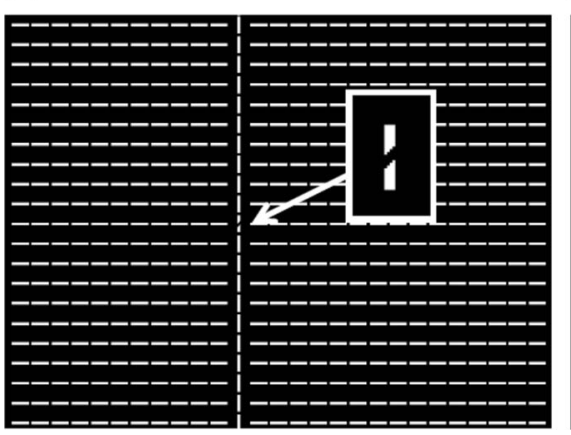

(B) Non-collinear distractor

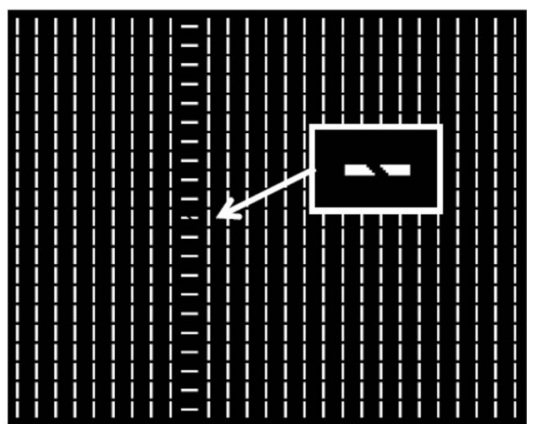

Fig. 1 a This is the condition in which the collinear distractor can mask a local target (enlarged at side). b An example of the display where the global distractor (noncollinear) facilitates the discrimination speed of the local target 
Humphreys, 2002; Han, Humphreys, \& Chen, 1999; Han, Jiang, Mao, Humphreys, \& Gu, 2005; Martin, 1979), in which it was found that a local target discrimination was delayed by a global structure, but not vice versa (Navon, 1977, 2003). For example, when small $E$ s were used to construct a large $F$, it took longer to discriminate the local $E$ s when compared to the condition where the small $E$ s were arranged into a large $E$. As well, global letter discrimination was found to be independent of its local letter form. Han and Humphreys (2002) proposed that this global interference was because the global structure was more salient than a local item, and attention selected the most salient item to process before the less salient items. In their experiments, they showed that when a local $E$ was red among other black local $E$ s, discrimination of such a salient local target was hindered by the shape of the global structure. In a similar way our local target (see Fig. 1) is not perceptually distinct. By increasing its salience to make it the first sighted item in the display, the influence of the global distractor might disappear.

On the other hand, reduction of the salience of the global structure might also lower the global interference effect (Han \& Humphreys, 2002; Han et al., 1999; Martin, 1979). In that research, adding grids in the background reduced segmentation of the global structure from noise, leading to loss of the global interference effect. Similarly, if we reduce the salience of the global collinear distractor in our search display, the competition may favor the local target to the extent that the collinear masking effect disappears.

\section{Design implementation}

In this study, we varied salience to boost the competitive advantage for the local target by making the target a color singleton (Experiment 1), a luminance singleton (Experiment 2), or an onset singleton (Experiment 3) in the search display. We reduced global structure salience by creating a heterogeneous background consisting of random orientation bars
(Experiment 4) or manipulating orientation of the flanking bars next to the collinear distractor (Experiment 5).

Our prediction is that, if the masking effect from the collinear distractor is mainly from local and global salience conflict, then increasing the salience of the local target or decreasing the salience of the global distractor would attenuate or eliminate the masking effect. If mechanisms other than local/global salience competition account for the target search impairment overlapping with the collinear distractor, our salience manipulations should have little effect on the masking effect.

The execution of our research relies on the success of our salience manipulations. To validate our salience manipulations, we included the search display with a noncollinear distractor (i.e., ladder shape; see Fig. 1b) as a sanity check. A noncollinear distractor facilitates, rather than impairs, visual searching (Jingling \& Tseng, 2013), which is in line with other attentional capture reports (Rauschenberger, 2003; Theeuwes, 2010; Turatto \& Galfano, 2000, 2001; Turatto et al., 2004). If we effectively increase the local target salience (Experiments 1-3) or reduce the distractor salience (Experiments 4-5), such target search facilitation effect should be less obvious since the target becomes more attractive and less affected by the background context.

\section{Experiment 1: The target as a color singleton}

\section{Method}

In this experiment, we compared a black tilted target (i.e., the tilted singleton used in our previous studies; e.g., Jingling \& Tseng, 2013) with a red tilted target to test whether the latter condition reduced the masking effect from the collinear distractor (see Fig. 2). The red target had more salience because it was a tilted and colored singleton. If it captured attention and was the first sighted stimulus in the display, then the orientation of the bars in the background should not affect the search speed.

\section{(A) Collinear distractor}

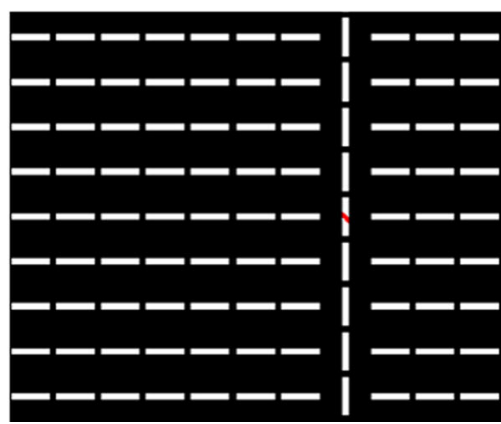

(B) Non-collinear distractor

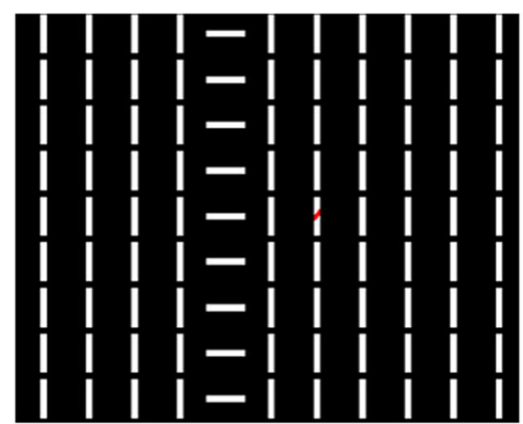

Fig. 2 Examples of the part of the search display with red targets. a left-tilted target overlapping with a collinear distractor. b A right-tilted target in the background, nonoverlapping with a noncollinear distractor 




Target color and distractor types

Fig. 3 Results in Experiment 1. Error bars are standard error of the mean.

\section{Participants}

We recruited 20 participants, and this number was estimated from our previous studies that showed that about 10 to 15 participants were enough to obtain statistical power for our interest (Chiu \& Jingling, 2014, 2015; Chow et al., 2013; Jingling, Tan, \& Tseng, 2013; Jingling \& Tseng, 2013; Tseng \& Jingling, 2015; Jingling, Tseng, \& Zhaoping, 2013). All the participants had normal or corrected-to-normal vision according to self-reports. None of them reported having color blindness, and all participants were given practice trials to ensure they were able to detect color targets. They each signed an informed consent form. We compensated their time with additional course credits.

\section{Apparatus and stimuli}

We carried out this experiment in a quiet, dimly lit room. We presented stimuli on a 17 -in. Dell monitor controlled by a dual-core CPU Acer desktop. The screen refresh rate was fixed at $85 \mathrm{~Hz}$, and the resolution was $1024 \times 768$ with a viewing distance of $60 \mathrm{~cm}$. The program used MATLAB with Psychophysics Toolbox Version 3 (Brainard, 1997; Pelli, 1997).

In the fixation display, a $0.18^{\circ} \times 0.18^{\circ}$ white square occupied the center of the screen, whereas the background was black. In the search display (see Fig. 2), a textured pattern was composed of $27 \times 21$ white $\left(65 \mathrm{~cd} / \mathrm{m}^{2}\right.$ and mean chromaticity at $[0.32,0.33]$ in CIE $1931-x y$ coordinates) bars against a black $\left(0.5 \mathrm{~cd} / \mathrm{m}^{2}\right.$ and mean chromaticity at $(0.34,0.33)$ in CIE $1931-x y$ coordinates) background. Each bar was $0.81^{\circ} \times 0.18^{\circ}$ in the visual angle, placed on a regular grid with unit grid spacing of $1.04^{\circ}$ both horizontally and vertically. The target was a small line with dimensions $0.63^{\circ} \times 0.11^{\circ}$ oriented either $45^{\circ}$ or $135^{\circ}$ from vertical and centered on a texture bar. In this experiment, the target was either red (mean luminance of $29.19 \mathrm{~cd} / \mathrm{m}^{2}$ and mean chromaticity at $[0.55,0.37]$ in CIE 1931-xy coordinates) or black.

The bars were all of the same orientation except in one column (i.e., distractor). There were two types of distractors. One had vertical bars, and the background columns were all horizontal to it (see Fig. 2a). The bars within the distractor column were collinear to each other and called the collinear distractor. The other distractor, called the noncollinear distractor, consisted of horizontal bars forming a ladder shape (see Fig. 2b).

\section{Design and procedure}

The target location was in the central (11th) row of one of the five possible columns (8th, 10th, 14th, 18th, and 20th) randomly selected in each trial. One of the (same) five columns was chosen in each trial as the distractor column, in which all element bars of the column were rotated by $90^{\circ}$ to form a salient structure. The distractor column was independently and randomly chosen so that only in one fifth of the trials did the target overlapped with the distractor column (namely, overlapping target).

There were three factors in this experiment: target color (red or black), target type (overlapping or nonoverlapping), and distractor type (collinear or noncollinear). 
Each participant completed 400 trials $(5$ Target Location $\times$ 5 Distractor Location $\times 2$ Target Color $\times 2$ Target Orientation [Left/Right] $\times 2$ Distractor Type $\times 2$ Repetition). Participants had 10 practice trials before data collection. During the experiment, a break was given after each 60 trials. Each trial began with a fixation display for $800 \mathrm{~ms}$, followed by the search display until the participant responded. After response, the next trial started immediately. We encouraged our participants to respond as quickly as possible without sacrificing accuracy. It took about 20 minutes to complete the task.

\section{Results}

To prevent outlier data influencing our conclusions, we excluded trials $(3.77 \%)$ longer than two standard deviations of the individual mean from subsequent analysis. Incorrect trials $(3.92 \%)$ were also excluded from the reaction time (RT) analysis. RT results are shown in Fig. 3 and accuracy results summarized in Table 1. We submitted the data to a three-way repeated-measures ANOVA with factors target color (black or red), target type (overlapping or nonoverlapping), and distractor type (collinear or noncollinear). RT and accuracy analysis results are summarized in Tables 2 and 3, respectively.

\section{Salience manipulation check}

Participants responded faster in trials with red targets than in those with black targets ( 701 vs. $788 \mathrm{~ms}), F(1,19)=23.12, p$ $=.0001, \eta_{\mathrm{p}}{ }^{2}=0.549$. The accuracy in trials with red $(93.5 \%)$ and black $(91.5 \%)$ color conditions was statistically equivalent, $F(1,19)=4.19, p=.055, \eta_{\mathrm{p}}{ }^{2}=0.148$, indicating no speed-accuracy trade-off. These results validated our salience manipulation, and red targets were indeed more salient than black targets so that the task became easier.

\section{Collinear masking effect}

The simple main effect analysis for the three-way interaction shows that the collinear masking effect appeared in displays with low color salience (black) and high color salience (red) targets, black target: 898 versus $723 \mathrm{~ms}, F(1,76)=59.78, p<$ $.0001, \eta_{\mathrm{p}}{ }^{2}=0.39$; red target: 749 versus $680 \mathrm{~ms}, F(1,76)=$ $9.35, p=.0031, \eta_{\mathrm{p}}{ }^{2}=0.06$. Accuracy analysis does not suggest a speed-accuracy trade off: the collinear masking effect was present for black targets, $84.46 \%$ versus $93.75 \%, F(1,76)$ $=36.46, p<.0001, \eta_{\mathrm{p}}{ }^{2}=0.478$, but not for red targets, $94.04 \%$ versus $94.12 \%, F(1,76)=.002, p=.96, \eta_{\mathrm{p}}{ }^{2}=0$ (see Fig. 3 ).

The collinear impairment effect was significantly larger for low color salience (black) targets than high salience (red) targets in both RT analysis, 175 versus $69 \mathrm{~ms}, F(1,38)=17.78, p$

Table 1 Accuracy (\%) summary table for all experiments

\begin{tabular}{|c|c|c|c|c|}
\hline & \multicolumn{2}{|c|}{ Collinear distractor } & \multicolumn{2}{|c|}{ Noncollinear distractor } \\
\hline & Nonoverlapping & Overlapping & Nonoverlapping & Overlapping \\
\hline \multicolumn{5}{|l|}{ Experiment 1} \\
\hline Black target & 93.75 & 84.46 & 90.17 & 97.75 \\
\hline Red target & 94.04 & 94.12 & 90.73 & 95.14 \\
\hline \multicolumn{5}{|l|}{ Experiment 2} \\
\hline Least salient target & 84.38 & 88.75 & 77.5 & 88.13 \\
\hline Middle salient target & 95.73 & 94.69 & 93.02 & 97.19 \\
\hline Most salient target & 98.33 & 95.94 & 95.73 & 97.19 \\
\hline \multicolumn{5}{|l|}{ Experiment 3} \\
\hline $0 \mathrm{~ms} \mathrm{SOA}$ & 95.59 & 90.44 & 84.41 & 94.19 \\
\hline $25 \mathrm{~ms} \mathrm{SOA}$ & 97.06 & 95.74 & 94.71 & 97.06 \\
\hline $50 \mathrm{~ms} \mathrm{SOA}$ & 98.53 & 96.62 & 96.18 & 96.54 \\
\hline $100 \mathrm{~ms} \mathrm{SOA}$ & 98.24 & 97.57 & 94.71 & 96.91 \\
\hline \multicolumn{5}{|l|}{ Experiment 4} \\
\hline Homogeneous background & 95.74 & 94.58 & 93.15 & 95.14 \\
\hline Heterogeneous background & 92.82 & 84.31 & 91.48 & 93.75 \\
\hline \multicolumn{5}{|l|}{ Experiment 5} \\
\hline Orthogonal flankers & 92.01 & 87.35 & - & - \\
\hline Parallel flankers & 95.19 & 91.37 & - & - \\
\hline Random flankers & 94.94 & 93.45 & - & - \\
\hline
\end{tabular}


Table 2 Reaction times ANOVA summary table for all experiments

\begin{tabular}{|c|c|c|c|c|}
\hline Name of effects & $F$ & $d f$ & $p$ & $\eta_{\mathrm{p}}^{2}$ \\
\hline \multicolumn{5}{|l|}{ Experiment 1} \\
\hline Target color (black, red) & 23.12 & 1,19 & 0.000 & 0.549 \\
\hline Target type (overlapping or nonoverlapping) & 19.67 & 1,19 & 0.000 & 0.509 \\
\hline Distractor type (collinear or non-collinear) & 14.70 & 1,19 & 0.001 & 0.436 \\
\hline Target Color $\times$ Target Type & 8.46 & 1,19 & 0.009 & 0.308 \\
\hline Target Color $\times$ Distractor Type & 2.09 & 1,19 & 0.169 & 0.099 \\
\hline Target Type $\times$ Distractor Type & 25.23 & 1,19 & 0.000 & 0.570 \\
\hline Three-way interaction & 10.83 & 1,19 & 0.004 & 0.363 \\
\hline \multicolumn{5}{|l|}{ Experiment 2} \\
\hline Target salience (most, middle, least) & 212.45 & 2,38 & 0.000 & 0.918 \\
\hline Target type (overlapping or nonoverlapping) & 7.47 & 1,19 & 0.013 & 0.282 \\
\hline Distractor type (collinear or noncollinear) & 0.04 & 1,19 & 0.843 & 0.002 \\
\hline Target Salience $\times$ Target Type & 1.86 & 2,38 & 0.197 & 0.089 \\
\hline Target Salience $\times$ Distractor Type & 11.43 & 2,38 & 0.000 & 0.376 \\
\hline Target Type $\times$ Distractor Type & 10.79 & 1,19 & 0.004 & 0.362 \\
\hline Three-way interaction & 2.08 & 2,38 & 0.014 & 0.099 \\
\hline \multicolumn{5}{|l|}{ Experiment 3} \\
\hline $\mathrm{SOA}(0,25,50,100 \mathrm{~ms})$ & 135.71 & 3,48 & 0.000 & 0.894 \\
\hline Target type (overlapping or nonoverlapping) & 14.19 & 1,16 & 0.002 & 0.470 \\
\hline Distractor type (collinear or noncollinear) & 3.40 & 1,16 & 0.084 & 0.175 \\
\hline SOA $\times$ Target Type & 0.32 & 3,48 & 0.814 & 0.019 \\
\hline SOA $\times$ Distractor Type & 0.96 & 3,48 & 0.424 & 0.056 \\
\hline Target Type $\times$ Distractor Type & 7.08 & 1,16 & 0.017 & 0.307 \\
\hline Three-way interaction & 7.20 & 3,48 & 0.000 & 0.310 \\
\hline \multicolumn{5}{|l|}{ Experiment 4} \\
\hline Background type (homo vs. hetero) & 21.15 & 1,28 & 0.000 & 0.416 \\
\hline Target type (overlapping or nonoverlapping) & 2.59 & 1,28 & 0.132 & 0.037 \\
\hline Distractor type (collinear or noncollinear) & 1.03 & 1,28 & 0.320 & 0.035 \\
\hline Background Type $\times$ Target Type & 2.49 & 1,28 & 0.137 & 0.059 \\
\hline Background Type $\times$ Distractor Type & 1.74 & 1,28 & 0.200 & 0.034 \\
\hline Target Type $\times$ Distractor Type & 38.65 & 1,28 & 0.000 & 0.558 \\
\hline Three-way interaction & 11.83 & 1,28 & 0.002 & 0.280 \\
\hline \multicolumn{5}{|l|}{ Experiment 5} \\
\hline Flanker type (orthogonal, parallel, random) & 5.18 & 2,26 & 0.013 & 0.285 \\
\hline Target type (overlapping or nonoverlapping) & 7.6 & 1,13 & 0.016 & 0.369 \\
\hline Flanker Type $\times$ Target Type & 1.99 & 2,26 & 0.157 & 0.133 \\
\hline
\end{tabular}

$=.0001, \eta_{\mathrm{p}}{ }^{2}=0.32$, and accuracy analysis, $9.29 \%$ versus $0.08 \%, F(1,38)=22.17, p<.0001, \eta_{\mathrm{p}}{ }^{2}=0.587$.

Therefore, local targets of higher color salience reduced, but not eliminated, the masking effect from the collinear distractor.

\section{Noncollinear facilitation effect (controlled comparison)}

In our controlled comparison, the results were consistent with the salience hypothesis: high color salience (red) targets received bigger search facilitation than low color salience (black) targets.

High color salience (red) targets overlapping with noncollinear distractors received the search advantage in both reaction time analysis, 660 versus $716 \mathrm{~ms}, F(1,76)=6.06, p=$ $.0161, \eta_{\mathrm{p}}{ }^{2}=0.04$, as well as accuracy analysis, $97.75 \%$ versus $90.17 \%, F(1,76)=8.25, p=.0053, \eta_{\mathrm{p}}{ }^{2}=0.021$. Low color salience (black) targets received no search advantage in reaction time analysis, 747 versus $785 \mathrm{~ms}, F(1,76)=2.83, p=$ $.121, \eta_{\mathrm{p}}{ }^{2}=.037$, but the accuracy was higher when targets overlapped with the non-collinear distractor, $95.14 \%$ versus $90.73 \%, F(1,76)=24.29, p=.0001, \eta_{\mathrm{p}}{ }^{2}=0.317$. Overall, we did not find evidence for speed-accuracy trade-off.

\section{Discussion}

In this experiment, we replicated the collinear masking effect in both high and low salience conditions, and found the more salient red target was insufficient to remove the masking effect. Note that the RTs for red targets were shorter than black targets, which supports the effectiveness of our salience manipulations that found that red targets were indeed more salient than black targets. The collinear masking effect in a low salience (black target) condition (175 ms) was significantly reduced compared to that in a high salience (red target) condition (69 ms), suggesting that further increasing target salience might overcome the masking effect. In Experiment 2, we manipulated the degree of perceptual salience by adjusting target luminance contrasts, which allowed us to explore a wider range of stimuli levels. 
Table 3 Accuracy ANOVA summary table for all experiments

\begin{tabular}{|c|c|c|c|c|}
\hline Name of effects & $F$ & $d f$ & $p$ & $\eta_{\mathrm{p}}^{2}$ \\
\hline \multicolumn{5}{|l|}{ Experiment 1} \\
\hline Target color (black, red) & 4.19 & 1,19 & 0.054 & 0.184 \\
\hline Target type (overlapping or non-overlapping) & 0.7 & 1,19 & 0.412 & 0.037 \\
\hline Distractor type (collinear or non-collinear) & 4.9 & 1,19 & 0.039 & 0.206 \\
\hline Target color X Target type & 8.86 & 1,19 & 0.008 & 0.323 \\
\hline Target color X Distractor type & 22.66 & 1,19 & 0 & 0.545 \\
\hline Target type X Distractor type & 40.96 & 1,19 & 0 & 0.683 \\
\hline Three-way interaction & 13.66 & 1,19 & 0.002 & 0.415 \\
\hline \multicolumn{5}{|l|}{ Experiment 2} \\
\hline Target salience (most, middle, least) & 42.79 & 2,38 & 0 & 0.692 \\
\hline Target type (overlapping or non-overlapping) & 8.72 & 1,19 & 0.008 & 0.314 \\
\hline Distractor type (collinear or non-collinear) & 13.5 & 1,19 & 0.002 & 0.424 \\
\hline Target salience X Target type & 5.12 & 2,38 & 0.011 & 0.208 \\
\hline Target salience X Distractor type & 11.37 & 2,38 & 0 & 0.375 \\
\hline Target type X Distractor type & 15.71 & 1,19 & 0.001 & 0.453 \\
\hline Three-way interaction & 0.29 & 2,38 & 0.747 & 0.011 \\
\hline \multicolumn{5}{|l|}{ Experiment 3} \\
\hline $\operatorname{SOA}(0,25,50,100 \mathrm{~ms})$ & 30.47 & 3,48 & 0.000 & 0.657 \\
\hline Target type (overlapping or nonoverlapping) & 0.99 & 1,16 & 0.335 & 0.052 \\
\hline Distractor type (collinear or noncollinear) & 13.91 & 1,16 & 0.002 & 0.462 \\
\hline SOA $\times$ Target Type & 1.46 & 3,48 & 0.265 & 0.082 \\
\hline SOA $\times$ Distractor Type & 2.08 & 3,48 & 0.115 & 0.118 \\
\hline Target Type $\times$ Distractor Type & 17.76 & 1,16 & 0.001 & 0.526 \\
\hline Three-way interaction & 10.97 & 3,48 & 0.000 & 0.407 \\
\hline \multicolumn{5}{|l|}{ Experiment 4} \\
\hline Background type (homo vs. hetero) & 12.39 & 1,28 & 0.002 & 0.276 \\
\hline Target type (overlapping or nonoverlapping) & 3.25 & 1,28 & 0.082 & 0.067 \\
\hline Distractor type (collinear or noncollinear) & 2.93 & 1,28 & 0.097 & 0.096 \\
\hline Background Type $\times$ Target Type & 5.75 & 1,28 & 0.023 & 0.136 \\
\hline Background Type $\times$ Distractor Type & 4.83 & 1,28 & 0.036 & 0.105 \\
\hline Target Type $\times$ Distractor Type & 21.49 & 1,28 & 0.000 & 0.405 \\
\hline Three-way interaction & 6.68 & 1,28 & 0.015 & 0.167 \\
\hline \multicolumn{5}{|l|}{ Experiment 5} \\
\hline Flanker type (orthogonal, parallel, random) & 11.23 & 2,26 & 0.000 & 0.464 \\
\hline Target type (overlapping or nonoverlapping) & 15.32 & 1,13 & 0.002 & 0.535 \\
\hline Flanker Type $\times$ Target Type & 2.04 & 2,26 & 0.150 & 0.143 \\
\hline
\end{tabular}

\section{Experiment 2: Salience level manipulation in luminance dimension}

We manipulated the degree of salience of the local target in this experiment by manipulating its brightness relative to the other bars in the display. We sampled three different levels of brightness in hopes of seeing different effects: When the local target was the most salient item, the distractor in the search display should play no role, and therefore, no masking or facilitation effect was expected. When the local target salience was reduced, then the dependence of the distractor should be observed. If, on the other hand, perceptual salience was not the main contributor to the masking or facilitation effect, these effects should persist regardless of target salience level.

\section{Method}

We recruited another 20 participants and presented stimuli on a 21-in. ViewSonic monitor controlled by a dual-core CPU Acer desktop. The screen refresh rate was fixed on $70 \mathrm{~Hz}$, and 
the resolution was $1024 \times 768$. The program used MATLAB with Psychophysics Toolbox 2.54. We kept the visual angle of the stimuli approximately similar to that in Experiment 1 by adjusting the viewing distance to $75 \mathrm{~cm}$.

The search display (see Fig. 4) was similar to that used in Experiment 1, except for the following changes. First, the bars in the display were gray (RGB level $128,34.9 \mathrm{~cd} / \mathrm{m}^{2}$ ) rather than white (RGB level $256,129.0 \mathrm{~cd} / \mathrm{m}^{2}$ ), and the background was set on dim gray (RGB level $13,3.7 \mathrm{~cd} / \mathrm{m}^{2}$ ) rather than black (RGB level $0,0.2 \mathrm{~cd} / \mathrm{m}^{2}$ ). The target was not a tilt break on a bar but was a selected bar with a different brightness level compared to the background bars. Participants were asked whether the target bar in this trial was brighter or darker than the bars in background. We replaced a small gap with a whole bar as the target in order to enlarge the manipulation effectincreasing size leads to more salient changes. Our previous study showed that this task change did not alter the masking and facilitation effect (Tseng \& Jingling, 2015). The target bar could be $\pm 64\left(80.7\right.$ or $\left.10.8 \mathrm{~cd} / \mathrm{m}^{2}\right), \pm 52\left(69.3\right.$ or $\left.13.0 \mathrm{~cd} / \mathrm{m}^{2}\right)$, or $\pm 38\left(57.7\right.$ or $\left.17.3 \mathrm{~cd} / \mathrm{m}^{2}\right)$ of the 128 levels, making it the most salient, less salient, and the least salient target. There were four possible columns (8th, 12th, 16th, and 20th) of the total 27 columns where the target or the distractor could be present. Each participant completed 394 trials.

\section{Results}

To avoid possible bias caused by outlier data, we excluded all trials that exceeded two standard deviations of individual means from further analysis. As a result, $5.18 \%$ of the trials were excluded. Also, we excluded the error trials (3.05\%) from the RT analysis. Figure 5 depicts the RT results, and Table 1 shows the accuracy results. We analyzed the selected RTs and accuracy with a three-way repeated-measures ANOVA on factor target salience (most, middle, least salient), target type (overlapping or nonoverlapping), and distractor type (collinear or noncollinear). The results of ANOVA on RT and on accuracy are shown in Tables 2 and 3, respectively.



Fig. 4 Part of the search display in Experiment 2
Salience manipulation check

Results (Fig. 5 and Tables 1 and 2) show a main effect of target salience in both response time analysis, $F(2,38)=212.45, p<$ $.0001, \eta_{\mathrm{p}}{ }^{2}=.918$ and accuracy analysis, $F(2,38)=42.79, p<$ $.0001, \eta_{\mathrm{p}}{ }^{2}=.445$. Post hoc Tukey tests show that participants responded significantly faster $(949 \mathrm{~ms})$ and more accurately $(96.80 \%)$ in the most salient condition but not in the least salient condition $(1,321 \mathrm{~ms}, 84.69 \%), p \mathrm{~s}<.01$. The responses were also faster and more accurate in the middle salient condition than $(1,058 \mathrm{~ms}, 95.16 \%)$ in the least salient condition, $p s<.01$. There is no indication of speed-accuracy trade-off, and these results validated our manipulation to use high luminance contrast to increase local target salience.

\section{Collinear masking effect}

As shown in Fig. 5, the simple main effect analysis of the three-way interaction revealed that in the most salient condition, the targets overlapping $(1,036 \mathrm{~ms})$ with the collinear distractor prolonged response time compared to non-overlapping targets $(887 \mathrm{~ms}), F(1,114)=10.84, p=$ $.001, \eta_{\mathrm{p}}^{2}=.095$. No collinear masking effect was found at the middle or the least salient conditions, $p \mathrm{~s}>.05$. Interestingly, there was an unexpected but significant facilitating effect in the least salient condition: the targets overlapping with a collinear distractor $(1,278 \mathrm{~ms})$ were discriminated faster by participants than the nonoverlapping targets $(1,377 \mathrm{~ms})$, $F(1,114)=4.81, p=.03, \eta_{\mathrm{p}}^{2}=.042$. Accuracy data showed no significant collinear masking effect or facilitation effect in the three saliency-level conditions, speaking against speedaccuracy trade-off. Altogether, we did not find evidence to support the salience competition hypothesis that increased salience would reduce or remove the collinear masking effect in the conditions with the most salient target.

\section{Noncollinear facilitation effect (controlled comparison)}

With a noncollinear distractor, participants responded to overlapping targets faster than nonoverlapping targets (facilitation effect) at all target salience levels, $F(1,114)=4.37,10.35$, and $17.32, p=.04, .002$, and $<.0001, \eta_{\mathrm{p}}{ }^{2}=.038, .091$, and .152 , for the most, middle, and least salient conditions, respectively. Accuracy analysis also showed a consistent noncollinear facilitation effect in the middle and the least salient conditions, $F(1,114)=5.46$ and 35.52, $p=.02$ and $<.0001, \eta_{\mathrm{p}}{ }^{2}=.047$ and .312 , while there was no such effect in the most salient condition, $p=.42$. To summarize, there was no speed-accuracy trade-off.

Moreover, the noncollinear facilitation effect was reduced as the salience level increased in accuracy analysis, $F(2,76)=$ $8.11 p=.001, \eta_{\mathrm{p}}^{2}=.212$, while the response times were statistically equivalent speaking against speed-accuracy 




Fig. 5 Results of Experiment 2. Response time as a function of local target saliency. Error bars are the standard error. * indicates significant differences between overlapping and nonoverlapping conditions, $p \mathrm{~s}<.05$

trade-off. This is consistent with the prediction of the salience competition hypothesis.

\section{Discussion}

In this experiment, we manipulated salience level on a luminance dimension of the local target. Results showed that even at the most salient level we tested, targets still suffered from the masking effect if placed with collinear distractors. Thus, we concluded that increasing the salience of the local target on a luminance dimension did not eliminate the masking effect from task-irrelevant global distractors.

One of the goals of Experiment 2 was to investigate how the masking or facilitation effects from the distractors varied when the target became more salient. The salience competition hypothesis predicted that a more salient target should be more independent of its location and the search less affected by whether or not it overlapped with the distractor. This hypothesis was only supported in a noncollinear condition as facilitation was attenuated with increasing salience levels, but was rejected in collinear trials as the collinear masking effect persisted at high local salience condition.

Interestingly, and puzzling at the same time, as the salience of the local target was reduced, the collinear masking effect turned into a facilitation effect (see Fig. 5). A closer look of the data (see Table 4) suggested that the facilitation effect was mainly from the brighter target trials $(1,402 \mathrm{~ms} / 82.08 \%$ vs. $1,140 \mathrm{~ms} / 92.5 \%)$ and not darker target trials $(1,361 \mathrm{~ms} /$ $86.67 \%$ vs. $1,455 \mathrm{~ms} / 85 \%$ ). Similar patterns also appeared in the noncollinear distractor condition: brighter target trials

Table 4 The RT (ms) and accuracy (\%) in Experiment 2 separating trials into that with a brighter or a darker target

\begin{tabular}{|c|c|c|c|c|c|c|}
\hline & \multicolumn{3}{|c|}{ Collinear distractor } & \multicolumn{3}{|c|}{ Noncollinear distractor } \\
\hline & Most salient & Middle salient & Least salient & Most salient & Middle salient & Least salient \\
\hline \multicolumn{7}{|l|}{ Brighter target } \\
\hline \multirow[t]{2}{*}{ Nonoverlapping } & 906 & 1,037 & 1,402 & 1,044 & 1,238 & 1,500 \\
\hline & $97.92 \%$ & $94.17 \%$ & $82.08 \%$ & $93.96 \%$ & $91.67 \%$ & $71.04 \%$ \\
\hline \multirow[t]{2}{*}{ Overlapping } & 1,014 & 1,043 & 1,140 & 883 & 932 & 1,162 \\
\hline & $92.50 \%$ & $94.38 \%$ & $92.50 \%$ & $95.63 \%$ & $95.63 \%$ & $91.25 \%$ \\
\hline \multicolumn{7}{|l|}{ Darker target } \\
\hline \multirow[t]{2}{*}{ Nonoverlapping } & 870 & 1,057 & 1,361 & 926 & 1,060 & 1,349 \\
\hline & $98.75 \%$ & $97.29 \%$ & $86.67 \%$ & $97.50 \%$ & $94.38 \%$ & $83.96 \%$ \\
\hline \multirow[t]{2}{*}{ Overlapping } & 1,056 & 1,043 & 1,455 & 896 & 1,066 & 1,312 \\
\hline & $99.38 \%$ & $95.00 \%$ & $85.00 \%$ & $98.75 \%$ & $98.75 \%$ & $85.00 \%$ \\
\hline
\end{tabular}


caused larger search facilitation $(1,500 \mathrm{~ms} / 71.04 \%$ vs. 1,162 $\mathrm{ms} / 91.25 \%)$ than did the darker target trials $(1,349 \mathrm{~ms} / 83.96 \%$ vs. $1,312 \mathrm{~ms} / 85 \%)$. The asymmetry was not anticipated. At our postexperiment interviews with participants, some participants reported that brighter targets were easier to detect than darker targets, and in some trials when they did not detect a brighter target, they would press the detection button for a darker target although they might not actually have detected the target. If a discrimination task (i.e., whether the odd item was brighter or darker than other bars) were transformed into a detection task (i.e., is there anything brighter than average), it could induce a "singleton detection" attentional control setting (Bacon \& Egeth, 1994) in which participants were in a selective mode responding to their primary detection goal (e.g., brighter target) only. This may be a good strategy particularly in a difficult task (i.e., least salient condition) and explain the asymmetric result pattern. This task-dependent attentional control settings hypothesis implies top-down goal influences, which is beyond the scope of current study, but an interesting line for further investigation.

Another possibility is that our luminance manipulations were ineffective in perceptual salience, e.g., the highest contrast local target is not perceptually more salient than the least salient target. Our participants did respond significantly faster in trials with the most salient target (i.e., highest contrast) than with least salient (i.e., lowest contrast) targets (see Table 1), which validated our luminance salience manipulation. However, it is still possible, although unlikely, that the salience variety did not reach the threshold to break the masking. In Experiment 3, we further investigated this possibility by introducing the most attentional capturing feature-abrupt onset (Jonides \& Yantis, 1988; Rauschenberger, 2003; Theeuwes, 2010; Yantis \& Jonides, 1984).

\section{Experiment 3: The target as an onset singleton}

This experiment added abrupt onset condition to the local target to maximize its attentional priority. Abrupt onset refers to presenting the target asynchronously to the other items in the search display (e.g., Yantis \& Jonides, 1984). It is a strong feature to capture attention in attentional capture literature (Rauschenberger, 2003; Theeuwes, 2010; Yantis and Jonides, 1984; Yantis \& Jonides, 1984) and is successful even in conditions when a unique color or shape fails to affect responses (Yantis \& Egeth, 1999; Yantis \& Gibson, 1994; Yantis \& Hillstrom, 1994). In this experiment, we presented the target after the search display was shown. If the asynchronicity between the display and the target were longer, the target was more attractive. We chose four stimulus-onset asynchronies (SOAs; 0, 25, 50, and $100 \mathrm{~ms}$ ). When SOA was zero $\mathrm{ms}$, it was the original search condition used in our previous experiments in which the target and the search display were displayed simultaneously. We expected to observe both masking effect and the facilitation effect, in trials with collinear and noncollinear distractors. If, however, an SOA delay between search display and the subsequent onset target made the onset target the first priority to capture attention, both masking and facilitation effects would disappear. Otherwise, if these effects were still present, then it was likely that the effects were not due the competition for attentional priority between target and the distractor.

\section{Method}

Another 17 participants participated in Experiment 3. The search display was similar to that of the black target condition in Experiment 1, except that the target was presented after the search display had appeared. The SOAs between the display and the target were $0,25,50$, or $100 \mathrm{~ms}$. The procedure for these trials is illustrated in Fig. 6. Each trial started with a $500 \mathrm{~ms}$ fixation, and a background display appeared and stayed for the duration of its selected SOA before a target appeared in the display. A blank display was shown after key press, and the next trial followed after $800 \mathrm{~ms}$. Each participant completed 800 trials. Other details were the same as Experiment 1.

\section{Results}

We excluded the error trials $(3.89 \%)$ and the trials in which responses were longer than two standard deviations of each individual mean $(0.6 \%)$. We analyzed the selected RTs and accuracy with a three-way ANOVA with factors onset SOA $(0,25,50$, or $100 \mathrm{~ms})$, target type (overlapping or not), and distractor type (collinear or not). We plotted the RT results in Fig. 7, and the accuracy results are summarized in Table 1. The ANOVA summary is listed in Table 2 (RT) and Table 3 (Accuracy). The main effect of SOA and target type are significant, as were interaction between target type and distractor type, and the three-way interaction.

\section{Salience manipulation check}

The main effect of SOA in RT analysis was significant, $F(3$, 48) $=135.71, p<.0001, \eta_{\mathrm{p}}{ }^{2}=.894$, and the post hoc analysis showed that the stationary target (SOA $0 \mathrm{~ms}$ ) took participants longer to discriminate $(707 \mathrm{~ms})$ than those with longer SOA $(590 \mathrm{~ms}, 587 \mathrm{~ms}$, and $572 \mathrm{~ms}$ for SOA conditions of $25 \mathrm{~ms}, 50$ $\mathrm{ms}$, and $100 \mathrm{~ms}$, respectively), $p \mathrm{~s}<.05$. Consistently, accuracy was significantly lower for concurrent presentation $(0 \mathrm{~ms}$ SOA, 91.16\%) compared to the $25 \mathrm{~ms}(96.14 \%), 50 \mathrm{~ms}$ $(96.97 \%)$, and $100 \mathrm{~ms}(98.86 \%) \mathrm{SOA}$ conditions, $F(3,48)=$ $30.47, p<.0001, \eta_{\mathrm{p}}{ }^{2}=.657$.

Thus, responses to an onset target were faster than that to a nononset target, which validated our salience manipulation. 
Fixation

Background only



$800 \mathrm{~ms}$

Fig. 6 The procedure of a trial in Experiment 3. The circle marks the location of the target but was not shown in the experiment

\section{Collinear masking effect}

Simple main effect analysis showed (see Fig. 7) that for the trials with collinear distractors, overlapping targets were processed more slowly than nonoverlapping targets in all SOA conditions, $F(1,128)=32.61,12.22,6.45$, and $3.94, p=$ $.0001, .0006, .0120$, and $.0489, \eta_{\mathrm{p}}{ }^{2}=.172, .064, .034$, and .027 , for SOAs $0,25,50$, and $100 \mathrm{~ms}$, respectively. Therefore, onset targets did not eliminate the masking effect of the collinear distractor.

To examine whether there was a speed-accuracy trade-off, our accuracy analysis revealed a significant interaction between distractor type and SOA, $F(1,16)=$ 17.76, $p=.0007, \eta_{\mathrm{p}}{ }^{2}=.526$ and three-way interaction, $F(3,48)=10.97, p<.0001, \eta_{\mathrm{p}}{ }^{2}=.407$. The simple main effect suggested that the accuracy for targets overlapping with collinear distractor $(84.41 \%)$ was lower than that for nonoverlapping targets $(94.19 \%)$ at $0 \mathrm{~ms} \mathrm{SOA}, F(1,128)$ $=39.06, p<.0001, \eta_{\mathrm{p}}{ }^{2}=.211$. We did not find evidence for speed-accuracy trade-off.

\section{(A) Collinear distractor}

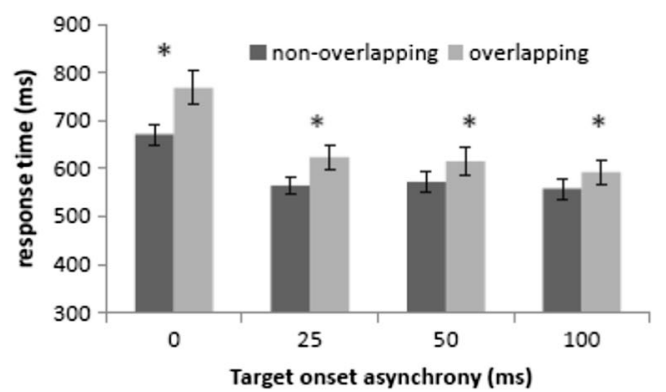

Noncollinear facilitation effect (controlled comparison)

Meanwhile, for trials with non-collinear distractors, search facilitation effects were not significant except at the lowest salience condition, 676 versus $710 \mathrm{~ms}, F(1,128)=3.98, p=.0478, \eta_{\mathrm{p}}{ }^{2}=$ $.029 ; 94.19 \%$ versus $84.41 \%, F(1,128)=10.82, p=.0013, \eta_{\mathrm{p}}{ }^{2}=$ .060. In other words, higher salient (onset) targets did eliminate the facilitation effect of the noncollinear distractor.

\section{Discussion}

This experiment enhanced the local target salience by introducing a temporal delay between the search display and the presence of the local target (i.e., making it a more salient onset target). In general, the simultaneous condition (0 ms) SOA replicated the previous observations (Chiu \& Jingling, 2014, 2015; Chow et al., 2013; Jingling, Tan, \& Tseng, 2013; Jingling \& Tseng, 2013; Jingling, Tseng, \& Zhaoping, 2013; Tseng \& Jingling, 2015) that the collinear distractor masked the local target, whereas the noncollinear distractor facilitated

(B) Non-collinear distractor

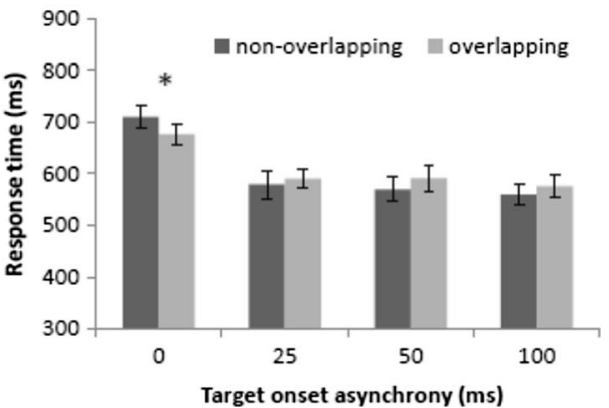

Fig. 7 The results of Experiment 3. Error bars are the standard error. a The collinear condition and $\mathbf{b}$ the noncollinear condition. * indicates significant differences between overlapping and nonoverlapping conditions, $p \mathrm{~s}<.05$ 
the local target. Nevertheless, when the target was presented abruptly as an onset, the facilitation effect by the noncollinear distractor disappeared. However, the masking effect by the collinear distractor still persisted. Thus, the facilitation effect by the noncollinear distractor might be a result of distractor salience and the masking effect by the collinear distractor to involve some other mechanisms than perceptual salience.

Experiments 1-3 together suggest that local target salience manipulations do not seem to alter the collinear masking effect. In the next two experiments, we adjusted the global distractor salience to examine the salience competition hypothesis.

\section{Experiment 4: Randomization background orientations bars}

The goal of this study was to test whether the background homogeneity affected the search performance. The more homogeneous the search background, the more salient the distractor. In this case, if the collinear distractor masked the search because of its super salience, then a less homogeneous background might reduce or eliminate the masking effect. The effect of the noncollinear distractor was included as well as control.

\section{Participants}

Each participant only performed one distractor type condition because of their time availability. To ensure each condition had at least 15 participants, we recruited 30 naïve participants for this experiment. All of them had normal or corrected-to-normal vision, and each signed an informed consent.

\section{Method}

Figure 8 shows examples of the four possible search displays in this experiment. The four conditions were the combination of two distractor types (collinear/noncollinear) and background
(A)

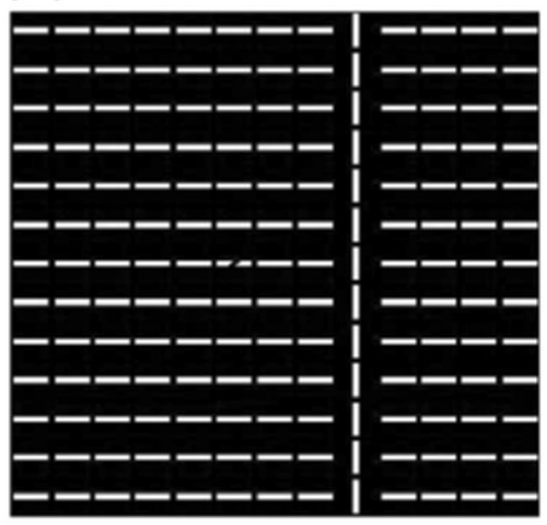

(C)



Fig. 8 Part of the search display used in Experiment 4. a The display with a collinear distractor in a homogeneous background; $\mathbf{b}$ a noncollinear distractor in a homogeneous background; $\mathbf{c}$ a collinear
(B)

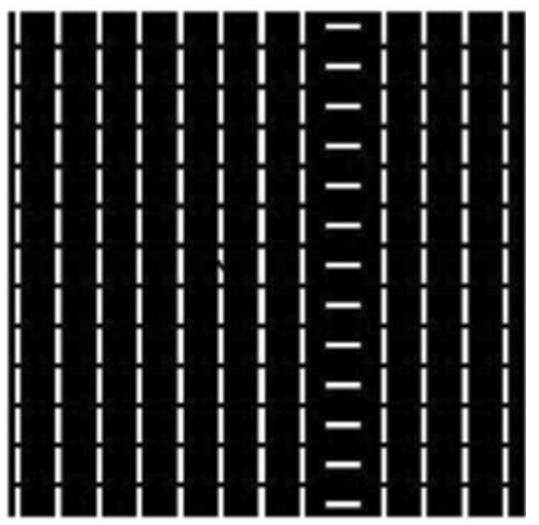

(D)

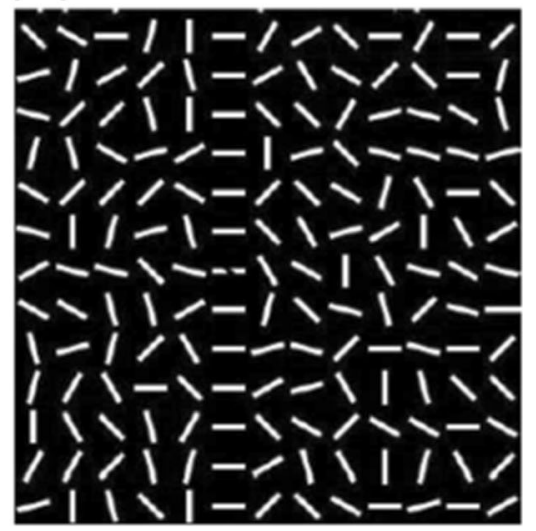

distractor in a heterogeneous background; and $\mathbf{d}$ a noncollinear distractor in a heterogeneous background 
types (homogeneous/heterogeneous). The length of the distractor was modified to 13 bars as any length more than five was established as enough to generate the collinear masking effect and noncollinear facilitation effect (Chow et al., 2013; Jingling \& Tseng, 2013). In the homogeneous background condition, all the bars not on the distractor were homogeneously oriented (see Fig. 8a, b). In the heterogeneous background condition (see Fig. 8c, d), background bars were randomly selected from 12 possible orientations $\left(0^{\circ}, 15^{\circ}, 30^{\circ}, 45^{\circ}, 60^{\circ}, 75^{\circ}, 90^{\circ}\right.$, $105^{\circ}, 120^{\circ}, 135^{\circ}, 150^{\circ}$, and $165^{\circ}$ ). To avoid unwanted groupings other than the distractor, the orientations within any $3 \times 3$ block that were repeated three times were replaced by another orientation. Other details were the same as that in the black target condition in Experiment 1. Three factors were manipulated in this study: target type (overlapping or not), distractor type (collinear or not), and background type (homogeneous or heterogeneous). The distractor type factor was a between factor, and each distractor type condition included 15 participants. The target and background factors types were within-factors. Each participant completed 392 trials.

\section{Results}

Inaccurate trials $(2.30 \%)$ or trials with response time greater than three standard deviations $(4.75 \%)$ were excluded from further RT analysis. RT results are depicted in Fig. 9, and the accuracy results summarized in Table 1. A mixed three-way ANOVA was carried out for RTs (Table 2) and accuracy (Table 3), and the factors were one between-factor (distractor type) and two within-factors (target and background types).

\section{Salience manipulation check}

Results show that the main effect of background types was significant, suggesting that responses were significantly faster and more accurate for trials with a homogeneous background (902 ms, 94.65\%) than those with a heterogeneous background $(1,009 \mathrm{~ms}, 90.59 \%), F(1,28)=21.15, p<.0001$, $\eta_{\mathrm{p}}{ }^{2}=.416$ (response time analysis) and $F(1,28)=12.39, p$ $=.0015, \eta_{\mathrm{p}}{ }^{2}=.276$ (accuracy analysis). Together, this supports the homogeneous background condition as an easier search condition.

\section{Collinear masking effect}

There was a significant interaction between target type and distractor type, $F(1,28)=38.65, p<.0001, \eta_{\mathrm{p}}{ }^{2}=.558$ as well as a three-way interaction, $F(1,28)=11.83, p=.0018, \eta_{\mathrm{p}}{ }^{2}=$ .280. The simple main effect analysis showed that, as illustrated in Fig. 9, trials with targets overlapping with collinear distracters required longer RTs by participants than nonoverlapping targets, both in a homogeneous background, $F(1,56)=$ $29.92, p<.0001, \eta_{\mathrm{p}}{ }^{2}=.238$, and a heterogeneous background $F(1,56)=17.48, p<.0001, \eta_{\mathrm{p}}{ }^{2}=.139$. Therefore, a collinear distractor impairs search in both types of backgrounds.

Accuracy impairment for targets overlapping with collinear distractors was reported in heterogeneous backgrounds, 84.31 versus $92.82 \%, F(1,56)=32.67, p<.0001, \eta_{\mathrm{p}}^{2}=.348$, only. In homogeneous backgrounds, there was no significant difference ( 94.58 vs. $95.74 \%$ ), possibly due to the ceiling effect. All together, we did not find evidence for speed-accuracy trade-off.

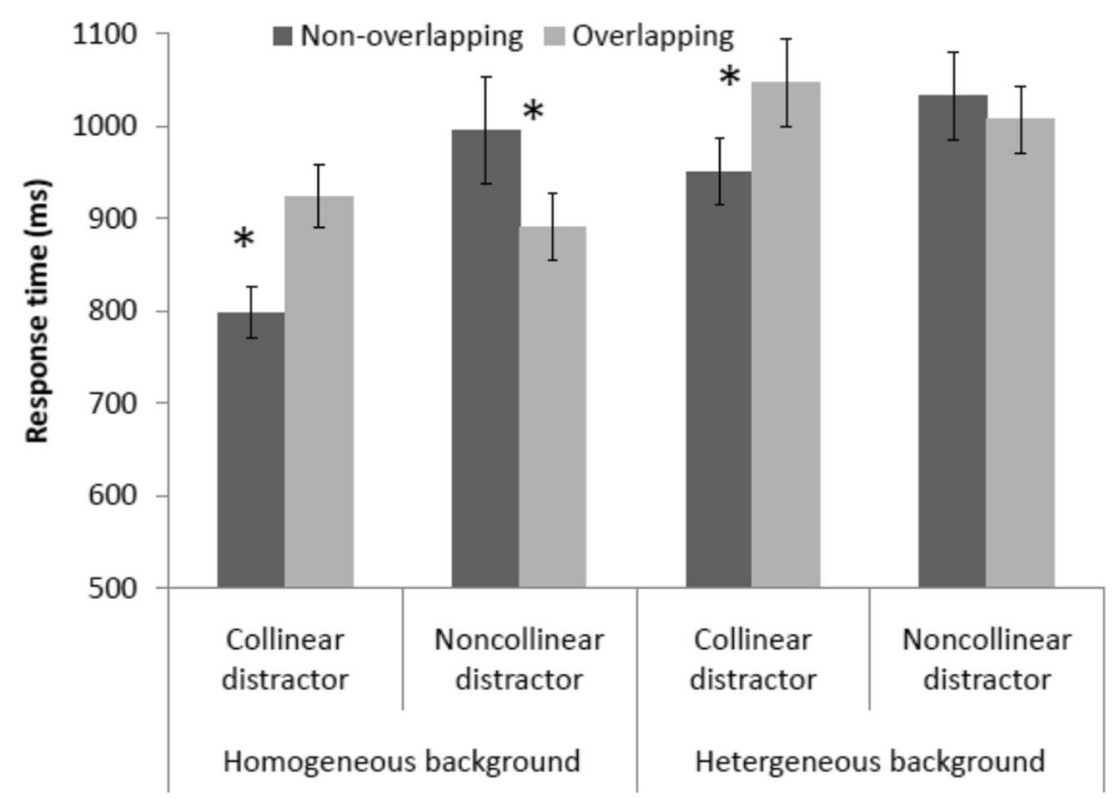

Fig. 9 The results of Experiment 4. Error bars are the standard error of the mean. * indicates significant differences between overlapping and nonoverlapping conditions, $p \mathrm{~s}<.05$ 


\section{Noncollinear facilitation effect (controlled comparison)}

When a distractor was non-collinear, search facilitation was only observed in a homogeneous background condition, $F(1$, 56) $=20.95, p<.0001, \eta_{\mathrm{p}}^{2}=.167$, not in heterogeneous background condition where the global distractor salience was reduced, $F(1,56)=1.22, p=.274, \eta_{\mathrm{p}}^{2}=.022$. Accuracy analysis did not reveal any significant differences.

\section{Discussion}

In this experiment, we decreased the salience of the distractor by placing it in a heterogeneous background. Results showed that collinear distractors were unaffective and still masked the local target, which prompted us to conclude that global and local salience competition was not a main contributor to the collinear masking effect. The noncollinear distractors, however, lost their search advantage as expected.

The salience computation of an item (Itti \& Koch, 2001; Li, 2002; Zhaoping, 2014) usually includes feature contrast values of the item with its neighboring items. To further understand whether the distractor salience is independent of the masking effect of the target, in the next experiment we manipulated the orientation contrast between the bars on the collinear distractor and its immediate neighboring bars (the flankers) of the distractor. In this case, flanking bars with 90-degree orientation contrast (see Fig. 10a) generated larger salience than flanking bars with parallel orientation (Fig. 10b) or random orientation (Fig. 10c). Similar manipulations can be found in Dakin and Baruch (2009). If distractor salience is critical for the collinear masking effect, we would expect to see varied effect size. As suggested by the previous four

(A)

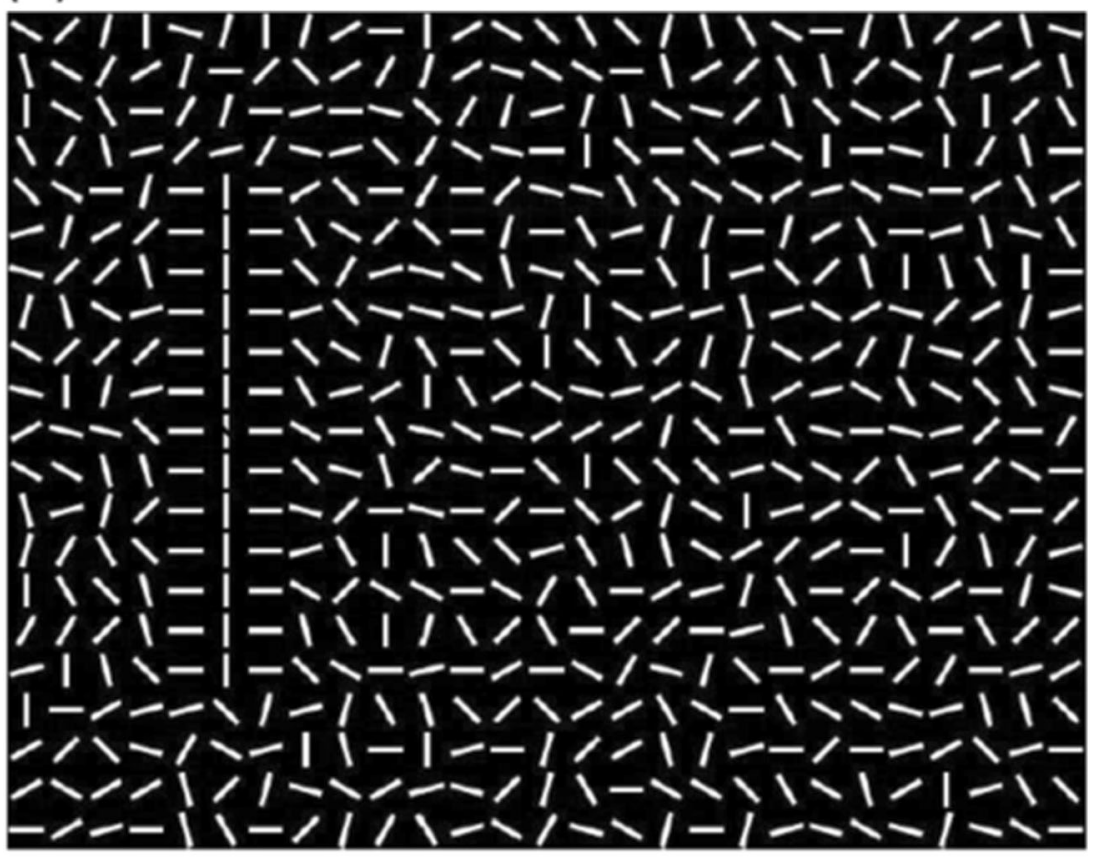

(B)

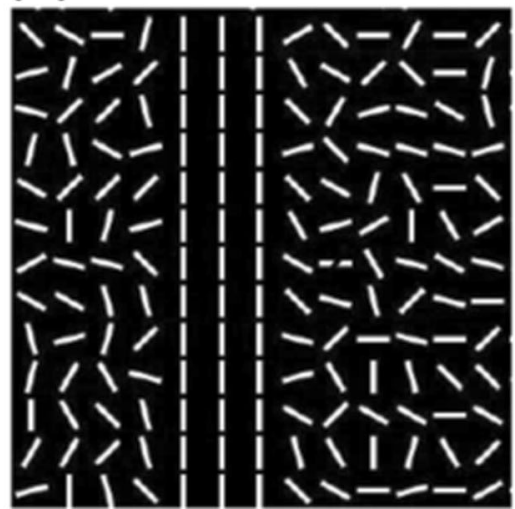

(C)

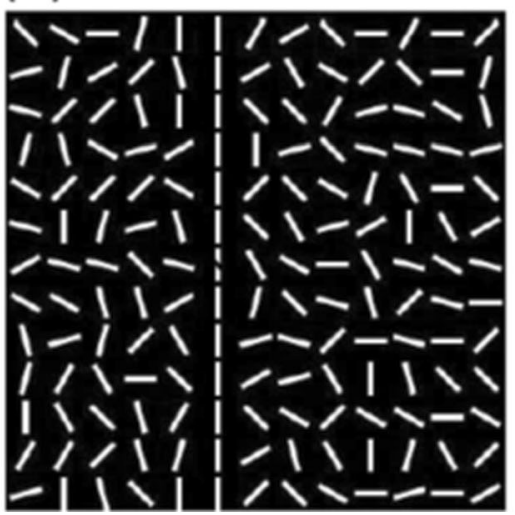

Fig. 10 The stimuli used in the Experiment 5. a orthogonal condition, b parallel condition, and $\mathbf{c}$ random condition 
experiments that the perceptual salience did not seem to be a major factor, then the collinear impairment masking effect should be observed regardless of the orientation contrasts of the flankers.

\section{Experiment 5: Flanker orientation manipulations on a heterogeneous background}

\section{Method}

Figure 10 summarized three types of flankers used in this experiment: the orthogonal flankers (see Fig. 10a) put horizontal bars next to the distractor; the parallel flankers (Fig. 10b) put vertical bars next to the distractor; and the random flankers (Fig. 10c) put bars in various orientations next to the distractor. The target could overlap with the distractor ( $25 \%$ of trials) or not ( $75 \%$ of trials). The four possible locations for target and distractor were randomly and independently determined (i.e., overlapping or not was task irrelevant). Sixteen naïve participants who had not participated in the previous experiments were recruited, and 10 practice trials were introduced before data collection. There were 192 trials for each type of search display, which constituted a total of 576 trials. The three flanker types were presented in mixed sequence with a break given every 64 trials. It took about an hour to complete this experiment. Other details were the same as those used in Experiment 4.

\section{Results}

Two participants' data were excluded from further analysis for their overall low accuracy ( $74.83 \%$ and $47.92 \%$, respectively) compared to the general performance $(96.33 \%)$. Trial RTs longer than two standard deviations of the mean $(3.11 \%)$ were also excluded. Figure 11 (RT) and Table 1 (accuracy) summarize the results. We submitted the data to a two-way repeated-measures ANOVA with factors target type (overlapping or nonoverlapping) and flanker type (orthogonal, parallel, and random), and the analysis results are available in Tables 2 and 3.

\section{Salience manipulation check}

Orthogonal flanker trials (highest salience) were significantly slower $(1,044 \mathrm{~ms})$ than random flankers trials (lowest salience, $1,008 \mathrm{~ms}), F(2,26)=5.17, p=.013, \eta_{\mathrm{p}}{ }^{2}=.285$. Also, orthogonal flanker trials (highest salience) had the lowest accuracy (89.68\%) compared to that of the other two types (93.28\% and $94.20 \%$ for parallel and random flankers, respectively), $F(2,26)=11.23, p=.0003, \eta_{\mathrm{p}}{ }^{2}=.464$, which validated our salience manipulation.

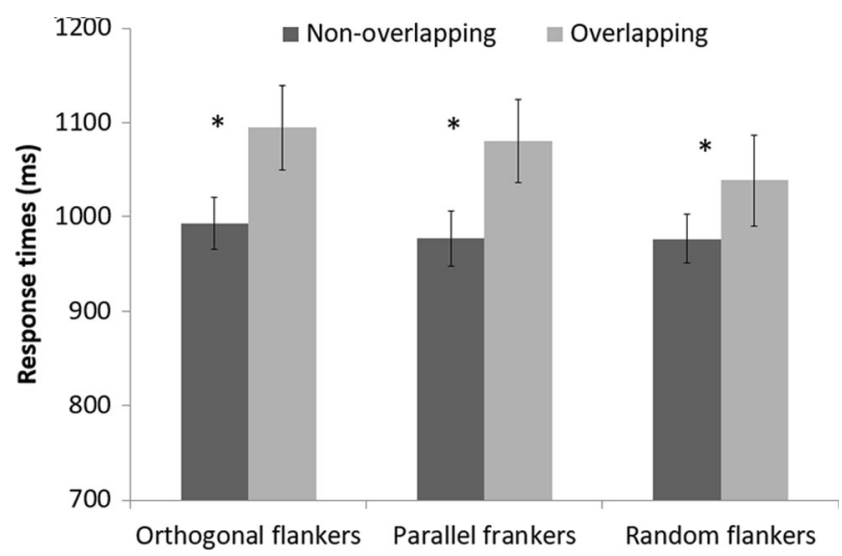

Fig. 11 The results of Experiment 5. Error bars are the standard error of the mean. * indicates significant differences between overlapping and nonoverlapping conditions, $p \mathrm{~s}<.05$. $*$ indicates significant differences between overlapping and nonoverlapping conditions, $p \mathrm{~s}<.05$

\section{Collinear masking effect}

Results show a significant main effect of target type in both RT, $F(1,13)=7.60, p=.0016, \eta_{\mathrm{p}}{ }^{2}=.369$ and accuracy analysis, $F(1,13)=15.32, p=.0018, \eta_{\mathrm{p}}{ }^{2}=.535$. The interaction between the target type and flanker type was not significant, $p \mathrm{~s}=.157$ and .150 for RT and accuracy, respectively. That is, trials with overlapping targets $(1,072 \mathrm{~ms})$ were slower and less accurate $(90.72 \%)$ than those with non-overlapping targets (982 ms, $94.05 \%$ ) in all flanker conditions. The orientation of the flankers did not modulate the size of collinear masking effect.

\section{Noncollinear facilitation effect (controlled comparison)}

Experiment 4 showed that the noncollinear distractor was unable to induce a facilitation effect with a heterogeneous search background. Thus in this experiment we did not carry out the noncollinear condition.

\section{Discussion}

In this experiment, the orientation contrasts between the collinear distractor and the flanking bars were manipulated. Theoretically the perceptual salience was higher for orthogonal flanker condition than for the random flanker condition, supported by the main effects in both RT and accuracy. However, the size of the collinear masking effect (the difference between overlapping and nonoverlapping targets) did not differ among flanker conditions (as indicated nonsignificant interaction). Therefore, we concluded that the collinear masking effect is not associated with perceptual salience of the distractor. 


\section{General discussion}

In this study, we investigated whether the local target masking effect by the collinear distractor was due to salience competition between the local target and global distractor. We increased local target salience (Experiments 1-3) and reduced global salience (Experiments 4-5), and the collinear masking effect was unaffected by salience change and persisted in all conditions. Although salience modulation seemed to affect the size of masking effect in Experiment 1, no similar sign was observed in other experiments. The masking effect present in all conditions was statistically equivalent in Experiment 3 (60, 43, and $34 \mathrm{~ms}$, respectively, in SOA 25, 50, and $100 \mathrm{~ms}$ conditions), $p=.362$, Experiment 4 (193 and $186 \mathrm{~ms}$ in the homogeneous and heterogeneous background), $p=.103$, and Experiment 5 (the global distractor salience level did not interact with the masking effect, $p=.157$ ). In Experiment 2, collinear masking only occurred in the highest salience condition. Meanwhile, a noncollinear global distractor facilitated search in the direction predicted by the salience competition hypothesis: it was most prominent (Experiment 2) or only occurred if the local target was of low salience (Experiments $1,3)$ and when the global distractor salience was high (Experiment 4). Altogether, we concluded that the masking effect induced by a collinear distractor was independent of perceptual salience and the facilitation effect from a noncollinear distractor might involve salience competition and operate differently from the collinear distractor.

The major result of this study rejected the conjecture that global/local salience competition is a main contributor to the collinear masking effect. Although collinear grouping increases the salience value in the orientation map (Jingling \& Zhaoping, 2008; Zhaoping, 2003), such an increment is not the reason collinear masking occurs. Current findings align with other research suggesting that the underlying process of this masking effect was unlikely governed by the salience map output: when salient global distractors were defined by color or luminance, they did not mask a local target but instead facilitated search (Jingling, Tseng, \& Zhaoping, 2013). Because a salience map is a combined representation of feature strengths from all dimensions, a process operated solely based on this map is responsive to the final combined salience value and should be blind to contributing feature sources. Therefore, distinct result patterns from the same global structure defined by different basic features (e.g., orientation, color, luminance) imply that participants have access to basic feature information and additional computation beyond feature contrast salience are involved. In summary, not all "super salient" global structures mask local targets, and the stimuli organization, such as collinear grouping, matters.

Our data also suggest that the collinear masking effect does not share the same mechanisms as the global interference effect, but the noncollinear facilitation effect might. Initially, the global interference effect appeared to occur when a global structure was processed preceding a local element (Navon, 1977, 2003). Han and Humphreys (2002), however, suggested that the global structure preceded the local structure because the former was more salient than the latter. In other words, perceptual salience, rather than global/local distinction, determines the global interference effect. Our manipulation of target salience or distractor salience did affect the manifestation of the facilitation effect from the noncollinear distractor. Our data suggest that the facilitation effect for local target search overlapping with non-collinear distractors may share the same mechanism as the global interference effect and could be removed when the local target was abruptly presented (Experiment 3), or when the noncollinear distractor was not salient enough (Experiment 4). In contrast to the noncollinear distractor, perceptual salience cannot cancel the collinear masking effect: the stimuli properties relating to collinear grouping play a more significant role.

There are several candidates that may account for the collinear masking effect. First, perceptual grouping of good continuity or contour integration (Field, Hayes, \& Hess, 1993; Hess et al., 2003) may matter. For instance, Kovács and Julesz (1993) showed that the detection threshold of a local target was elevated if the target was on the edge of a contour (see also Kovács, 1996; Kovács \& Julesz, 1994). Also, a snake contour is more salient and processed faster than a ladder contour (Dakin \& Baruch, 2009; Schmidt \& Vancleef, 2016; Vancleef \& Wagemans, 2013). Contour integration may integrate bars and thus make target discrimination more difficult. Second, visual crowding (e.g., Pelli, Palomares, \& Majaj, 2004) may also play a role. In particular, a snake contour produces a larger crowding effect than a ladder contour by using Gabor stimuli (Yeotikar, Khuu, Asper, \& Suttle, 2011). A ladder contour is not detectable in periphery (May $\&$ Hess, 2007), which also implies possible involvement of a crowding effect in contour integration. Our collinear distractor had smaller gaps between bars compared to those in the background, making visual crowding a possible source. Third, some processes relating to edge formation may also play a role. In particular, this effect is limited in display when the collinear column is orthogonal to the background bars (Jingling, Tseng, \& Zhaoping, 2013) and constructs a T junction between the collinear distractor and the background. Such $\mathrm{T}$ junctions are signals of object borders (Craft, Schütze, Niebur, \& Von Der Heydt, 2007; Qiu \& Von Der Heydt, 2005; Zhou, Friedman, \& Von Der Heydt, 2000) and could mask a local target on top of the border. Other low-level stimulus properties, such as perceptual filling-in (Zhaoping \& Jingling, 2008), may also be likely candidates.

In conclusion, the collinear masking effect was not induced by salience competition between the global distractor and the local target. After increasing perceptual salience of the local target, the collinear masking effect persisted and the 
facilitation effect by the noncollinear distractor disappeared. Understanding the underlying mechanism of the collinear masking effect gives us more knowledge on how visual search is more efficient and how we can hide unwanted items effectively.

Acknowledgements This work was supported by Grant MOST1032628-H-039-001-MY3 to L.J. and by Grant-in-Aid for Scientific Research (KAKENHI) from Japan Society for Promotion of Science to C.-h.T.

\section{References}

Bacon, W. F., \& Egeth, H. E. (1994). Overriding stimulus-driven attentional capture. Perception \& Psychophysics, 55(5), 485-496.

Brainard, D. H. (1997). The psychophysics toolbox. Spatial Vision, 10, 433-436.

Chiu, C.-W., \& Jingling, L. (2014). Focus size of attention: Exploring the focus hypothesis on the collinear masking effect in visual search. Chinese Journal of Psychology, 56(4), 467-484.

Chiu, C.-W., \& Jingling, L. (2015). Temporal characteristics of the collinear masking effect in visual search. Chinese Journal of Psychology, 57(1), 91-107.

Chow, H. M., Jingling, L., \& Tseng, C.-H. (2013). Collinear integration affects visual search at V1. Journal of Vision, 13(10), 1-20. doi:10. $1167 / 13.10 .24$

Chow, H. M., Jingling, L., \& Tseng, C.H. (2016). Eye of origin guides attention away: An ocular singleton column impairs visual search like a collinear column. Journal of Vision, 16(1), 12. doi:10.1167/16. 1.12

Conci, M., Müller, H. J., \& Elliott, M. A. (2007). Closure of salient regions determines search for a collinear target configuration. Perception \& Psychophysics, 69, 32-47.

Conci, M., Müller, H. J., \& von Mühlenen, A. (2013). Object-based implicit learning in visual search: Perceptual segmentation constrains contextual cueing. Journal of Vision, 13(3), 15. doi:10. $1167 / 13.3 .15$

Conci, M., \& von Mühlenen, A. (2009). Region segmentation and contextual cuing in visual search. Attention, Perception, \& Psychophysics, 71(1), 1514-1524.

Craft, E., Schütze, H., Niebur, E., \& Von der Heydt, R. (2007). A neural model of figure-ground organization. Journal of Neurophysiology, 97(6), 4310-4326.

Dakin, S. C., \& Baruch, N. J. (2009). Context influences contour integration. Journal of Vision, 13(2), 13.1-13. doi:10.1167/9.2.13

Field, D. J., Hayes, A., \& Hess, R. F. (1993). Contour integration by the human visual system: Evidence for a local "association field.". Vision Research, 33(2), 173-193.

Han, S., \& Humphreys, G. W. (2002). Segmentation and selection contribute to local processing in hierarchical analysis. The Quarterly Journal of Experimental Psychology: Section A, 55(1), 5-21.

Han, S., Humphreys, G. W., \& Chen, L. (1999). Parallel and competitive processes in hierarchical analysis: Perceptual grouping and encoding of closure. Journal of Experimental Psychology: Human Perception and Performance, 25(5), 1411.

Han, S., Jiang, Y., Mao, L., Humphreys, G. W., \& Gu, H. (2005). Attentional modulation of perceptual grouping in human visual cortex: Functional MRI studies. Human Brain Mapping, 25(4), 424-432.

Hess, R. F., Hayes, A., \& Field, D. J. (2003). Contour integration and cortical processing. Journal of Physiology-Paris, 97(2), 105-119.

Itti, L., \& Koch, C. (2001). Computational modelling of visual attention. Nature Reviews Neuroscience, 2(3), 194-203
Jingling, L., Tan, D.-L., \& Tseng, C.-H. (2013). Salient collinear grouping diminishes local salience in visual search: An eye movement study. Journal of Vision, 13(12), 1-10.

Jingling, L., \& Tseng, C.-H. (2013). Collinearity impairs local element visual search. Journal of Experimental Psychology: Human Perception and Performance, 39(1), 156-167. doi:10.1037/ a0027325

Jingling, L., Tseng, C.-H., \& Zhaoping, L. (2013). Orientation is different: Interaction between contour integration and feature contrasts in visual search. Journal of Vision, 13(3), 1-13.

Jingling, L., \& Zhaoping, L. (2008). Change detection is easier at texture border bars when they are parallel to the border: Evidence for V1 mechanisms of bottom-up salience. Perception, 37(2), 197-206.

Jonides, J., \& Yantis, S. (1988). Uniqueness of abrupt visual onset in capturing attention. Perception \& Psychophysics, 43(4), 346-354.

Kimchi, R., Yeshurun, Y., Spehar, B., \& Pirkner, Y. (2016). Perceptual organization, visual attention, and objecthood. Vision Research, 126, 34-51.

Kimchi, R., Yeshurun, Y., \& Cohen-Savransky, A. (2007). Automatic, stimulus-driven attentional capture by objecthood. Psychonomic Bulletin \& Review, 14(1), 166-172.

Kovács, I. (1996). Gestalten of today: Early processing of visual contours and surfaces. Behavioural Brain Research, 82(1), 1-11.

Kovács, I., \& Julesz, B. (1993). A closed curve is much more than an incomplete one: Effect of closure in figure-ground segmentation. Proceedings of the National Academy of Sciences, 90(16), 7495-7497.

Kovács, I., \& Julesz, B. (1994). Perceptual sensitivity maps within globally defined visual shapes. Nature, 370(6491), 644-646.

Li, Z. (2002). A saliency map in primary visual cortex. Trends in Cognitive Sciences, 6(1), 9-16.

Martin, M. (1979). Local and global processing: The role of sparsity. Memory \& Cognition, 7(6), 476-484.

May, K. A., \& Hess, R. F. (2007). Ladder contours are undetectable in the periphery: A crowding effect? Journal of Vision, 7(9), 1-15.

Navon, D. (1977). Forest before trees: The precedence of global features in visual perception. Cognitive Psychology, 9, 353-383.

Navon, D. (2003). What does a compound letter tell the psychologist's mind? Acta Psychologica, 114, 273-309.

Pelli, D. G. (1997). The VideoToolbox software for visual psychophysics: Transforming numbers into movies. Spatial Vision, 10(4), 437-442.

Pelli, D. G., Palomares, M., \& Majaj, N. J. (2004). Crowding is unlike ordinary masking: Distinguishing feature integration from detection. Journal of Vision, 4(12), 1136-1169.

Qiu, F. T., \& Von Der Heydt, R. (2005). Figure and ground in the visual cortex: V2 combines stereoscopic cues with Gestalt rules. Neuron, 47(1), 155-166.

Rauschenberger, R. (2003). Attentional capture by auto-and allo-cues. Psychonomic Bulletin \& Review, 10(4), 814-842.

Schmidt, F., \& Vancleef, K. (2016). Response priming evidence for feedforward processing of snake contours but not of ladder contours and textures. Vision Research, 126, 174-182.

Theeuwes, J. (2010). Top-down and bottom-up control of visual selection. Acta Psychologica, 135(2), 77-99.

Tseng, C., \& Jingling, L. (2015). A salient and task-irrelevant collinear structure hurts visual search. PLOS ONE, 10(4), e0124190. doi:10. 1371/journal.pone. 0124190

Turatto, M., \& Galfano, G. (2000). Color, form and luminance capture attention in visual search. Vision Research, 40(13), 1639-1643.

Turatto, M., \& Galfano, G. (2001). Attentional capture by color without any relevant attentional set. Perception \& Psychophysics, 63(2), 286-297.

Turatto, M., Galfano, G., Gardini, S., \& Mascetti, G. G. (2004). Stimulusdriven attentional capture: An empirical comparison of display-size and distance methods. Quarterly Journal of Experimental Psychology Section A, 57(2), 297-324. 
Vancleef, K., \& Wagemans, J. (2013). Component processes in contour integration: A direct comparison between snakes and ladders in a detection and a shape discrimination task. Vision Research, 92, 39-46.

Wolfe, J. M. (2010). Visual search. Current Biology, 20(8), R346-R349.

Yantis, S., \& Egeth, H. E. (1999). On the distinction between visual salience and stimulus-driven attentional capture. Journal of Experimental Psychology: Human Perception and Performance, 25, 661-676.

Yantis, S., \& Gibson, B. S. (1994). Object continuity in apparent motion and attention. Canadian Journal of Experimental Psychology/Revue canadienne de psychologie expérimentale, 48, 182-204.

Yantis, S., \& Hillstrom, A. P. (1994). Stimulus-driven attentional capture: evidence from equiluminant visual objects. Journal of Experimental Psychology: Human Perception and Performance, 20, 95-107.

Yantis, S., \& Jonides, J. (1984). Abrupt visual onsets and selective attention: Evidence from visual search. Journal of Experimental Psychology: Human Perception and Performance, 10, 601-621.
Yeotikar, N. S., Khuu, S. K., Asper, L. J., \& Suttle, C. M. (2011). Configuration specificity of crowding in peripheral vision. Vision Research, 51(11), 1239-1248. doi:10.1016/j.visres.2011.03.016

Yeshurun, Y., Kimchi, R., Sha'shoua, G., \& Carmel, T. (2009). Perceptual objects capture attention. Vision Research, 49(10), 1329-1335.

Zhaoping, L. (2003). V1 mechanisms and some figure-ground and border effects. Journal of Physiology-Paris, 97(4), 503-515.

Zhaoping, L. (2014). Understanding vision: Theory, models, and data. Oxford: Oxford University Press.

Zhaoping, L., \& Jingling, L. (2008). Filling-in and suppression of visual perception from context: A Bayesian account of perceptual biases by contextual influences. PLOS Computational Biology, 4(2), e14.

Zhou, H., Friedman, H. S., \& Von Der Heydt, R. (2000). Coding of border ownership in monkey visual cortex. The Journal of Neuroscience, 20(17), 6594-6611. 